\title{
Impact of MR-Based Attenuation Correction on Neurologic PET Studies
}

\author{
Yi Su${ }^{1}$, Brian B. Rubin ${ }^{1}$, Jonathan McConathy ${ }^{1}$, Richard Laforest ${ }^{1}$, Jing Qi ${ }^{1}$, Akash Sharma ${ }^{1}$, Agus Priatna ${ }^{2}$, \\ and Tammie L.S. Benzinger ${ }^{1,3}$ \\ ${ }^{I}$ Mallinckrodt Institute of Radiology, Washington University School of Medicine, St. Louis, Missouri; ${ }^{2}$ Siemens Medical Solutions, \\ St. Louis, Missouri; and ${ }^{3}$ Department of Neurological Surgery, Washington University School of Medicine, St. Louis, Missouri
}

\begin{abstract}
Hybrid PET and MR scanners have become a reality in recent years, with the benefits of reduced radiation exposure, reduction of imaging time, and potential advantages in quantification. Appropriate attenuation correction remains a challenge. Biases in PET activity measurements were demonstrated using the current MRbased attenuation-correction technique. We aimed to investigate the impact of using a standard MR-based attenuation correction technique on the clinical and research utility of a PET/MR hybrid scanner for amyloid imaging. Methods: Florbetapir scans were obtained for 40 participants on a hybrid scanner with simultaneous MR acquisition. PET images were reconstructed using both MRand CT-derived attenuation maps. Quantitative analysis was performed for both datasets to assess the impact of MR-based attenuation correction to absolute PET activity measurements as well as target-to-reference ratio (SUVR). Clinical assessment was also performed by a nuclear medicine physician to determine amyloid status based on the criteria in the Food and Drug Administration prescribing information for florbetapir. Results: MR-based attenuation correction led to underestimation of PET activity for most parts of the brain, with a small overestimation for deep brain regions. There was also an overestimation of SUVRs with cerebellar reference. SUVR measurements obtained from the 2 attenuationcorrection methods were strongly correlated. Clinical assessment of amyloid status resulted in identical classification as positive or negative regardless of the attenuation-correction methods. Conclusion: MR-based attenuation correction causes biases in quantitative measurements. The biases may be accounted for by a linear model, although the spatial variation cannot be easily modeled. The quantitative differences, however, did not affect clinical assessment as positive or negative.
\end{abstract}

Key Words: PET/MR; attenuation correction; amyloid imaging

J Nucl Med 2016; 57:913-917

DOI: 10.2967/jnumed.115.164822

$\mathbf{P}_{\mathrm{B}}$ ET is a widely used molecular imaging modality that can be used to investigate various physiologic, functional, and molecular processes in vivo through targeted radiolabeled tracers (1). Meanwhile, MR imaging is known for its high spatial resolution and superb

Received Jul. 30, 2015; revision accepted Dec. 29, 2015.

For correspondence or reprints contact: Yi Su, Campus Box 8131, Mallinckrodt Institute of Radiology, Washington University School of Medicine, 510. S. Kingshighway Blvd., St. Louis, MO 63110.

E-mail: suy@mir.wustl.edu

Published online Jan. 28, 2016.

COPYRIGHT (c) 2016 by the Society of Nuclear Medicine and Molecular Imaging, Inc. soft-tissue contrast (2). Combining the capabilities of MR and PET imaging offers unique opportunities for both diagnostic and research purposes (3). The clinical advantages of a combined PET/MR system is an active area of investigation, and the potential benefits include but are not limited to reduced radiation exposure (4), MR-based motion correction (5), image-derived arterial input function (6), and a single imaging session for PET and MR acquisition in debilitated or cognitively impaired patients. Despite the technical difficulties in the development of a combined PET/MR system, tremendous effort has been devoted to make it a reality (2) for clinical whole-body imaging. One combines a time-of-flight PET together with a 3-T MR in a sequential design (7). The other is a fully integrated PET/MR system with a MR-compatible PET system mounted inside a 3-T MR system, so that it is capable of truly simultaneous acquisition of PET and MR (8). More recently, a combined PET/MR scanner with time-offlight capability was also developed (9).

Among the various technical challenges for an integrated PET/ MR system is the generation of an accurate attenuation map ( $\mu$-map) to account for the signal reduction when annihilation photons travel from the location of positron annihilation and the detectors remain an active area of research (10-12). In a traditional standalone PET system, transmission scans with radioactive source attached to the scanner can be acquired to directly measure the $\mu$-map (13). For a combined PET/CT system, a low-dose CT scan is obtained, and then the measured $\mu$-map for CT energy photon is converted to a PET $\mu$-map (14-16) for attenuation correction (AC). For hybrid PET/MR systems, traditional approaches to obtain a $\mu$-map are typically unavailable, and MR-based approaches are commonly used $(7,8)$. However, this is challenging because there is not a simple relationship between the MRI signal based on proton density and relaxation time and the required PET $\mu$-map depending on the local electron density. In the Siemens PET/MR system, a 2-point Dixon MR sequence is used to obtain images, the voxels of which are classified into air, lung, fat, and soft tissue. A corresponding linear attenuation coefficient is then assigned to each tissue class (8). A similar approach is taken by the simultaneous PET/MR system from GE Healthcare (Signa PET/MR) and the sequential PET/MR system from Philips (Ingenuity TF PET/MR) (7,9). In addition to these 2 approaches implemented on available clinical scanners, other experimental approaches are currently being investigated $(12,17)$. Several groups have performed theoretic or experimental studies to evaluate the performance of common MR-based AC methods (MRAC) $(18,19)$ and identified significant signal reduction close to or within bone. The magnitude of signal reduction can be as high as $10 \%-20 \%$ (20). However, the impact of the signal loss on specific clinical or research applications is still unclear. The goal of this article was to investigate the impact of using a standard MRAC technique on the 
clinical and research utility of PET/MR hybrid scanners for amyloid imaging.

\section{MATERIALS AND METHODS}

\section{Participants}

Forty participants were selected from an ongoing study at Knight Alzheimer Disease Research Center at Washington University School of Medicine to compare 2 amyloid imaging tracers: ${ }^{11} \mathrm{C}$-Pittsburgh compound $\mathrm{B}(\mathrm{PiB})(21)$ and ${ }^{18} \mathrm{~F}-$ florbetapir (AV45) (22). The cohort was intentionally enriched with high-amyloid-level cases to increase the chances of finding clinically positive scans based on the AV45 data so that we could assess the impact of AC on clinical diagnosis using florbetapir scans. The enrichment was achieved by identifying 20 participants with the highest $\mathrm{PiB}$ retention based on their mean cortical binding potential $(23,24)$ values. Then 20 additional participants were randomly selected from the rest of the enrolled participants who completed their imaging studies before October 2013. The cohort had a mean age of $69 \mathrm{y}$. One of the 40 participants had a clinical dementia rating (25) of 0.5 (very mild dementia) whereas the clinical dementia rating for the rest was 0 (cognitively normal). Twenty-two participants were considered $\mathrm{PiB}$-positive based on a mean cortical binding potential cutoff of 0.18 (cerebellar cortex referencing without partial-volume correction) (23,24). All assessment and imaging procedures were approved by Washington University's Human Research Protection Office. Written informed consent was obtained from all individuals or their care givers.

\section{Imaging Acquisition}

Each participant underwent 2 separate imaging sessions within $2 \mathrm{wk}$ of each other. ${ }^{18} \mathrm{~F}$-florbetapir scanning was performed on a Biograph mMR PET/MR hybrid scanner (Siemens) with approximately $370 \mathrm{MBq}$ of tracer. Emission data were collected in list-mode either starting at tracer injection for $70 \mathrm{~min}$ or starting $50 \mathrm{~min}$ after injection for $20 \mathrm{~min}$. The standard Dixon sequence on the scanner was collected at the beginning of scanning for MRAC (8). T1-weighted images were acquired using a sagittal magnetization-prepared rapid gradient echo (MPRAGE) sequence with the following imaging parameters: repetition time/echo time, 2,300/2.95 ms; inversion time, $900 \mathrm{~ms}$; flip angle, $9^{\circ}$; and voxel size, $1.1 \times 1.1 \times 1.2 \mathrm{~mm}$. PiB scanning was performed on a Biograph 40 PET/CT scanner (Siemens) with approximately $444 \mathrm{MBq}$ of tracer with CT-based AC (CTAC). Only the CT portion of the PiB scan was used in this work. CT images of the head were acquired at 120 $\mathrm{keV}, 25$ effective mAs with a voxel size of $0.59 \times 0.59 \times 3.0 \mathrm{~mm}$ and a matrix size of $512 \times 512 \times 74$.

\section{Image Reconstruction}

PET image reconstruction was performed offline using e7tools (Siemens). For each participant, only the portion of list-mode data collected between 50 and $70 \mathrm{~min}$ after injection was used for reconstruction regardless of the acquisition protocol. An ordered-subset expectation maximization algorithm with 3 iterations and 21 subsets was used with a matrix size of $256 \times 256 \times 127$ and a voxel size of $1.4 \times 1.4 \times$ $2.0 \mathrm{~mm}$. Standard normalization, dead time, random, and scatter correction (with relative scaling) were performed using the default settings of the e7tools. Postreconstruction filtering was also performed with $4 \mathrm{~mm}$ in full width at half maximum 3-dimensional isotropic gaussian filter. AC was performed with both a Dixon-based $\mu$-map and a CT-based $\mu$-map. For Dixon-based MRAC, the $\mu$-maps exported from the mMR scanner were used. For CTAC, the CT images were coregistered with the MPRAGE and Dixon MR using a vector gradient algorithm (26). For voxels within the CT field of view, attenuation coefficients were estimated by converting CT Hounsfield values into attenuation coefficients for $511-\mathrm{keV}$ photons using a bilinear function defined by 3 control points: $\left(-1,000 \mathrm{HU}, 0 \mathrm{~cm}^{-1}\right),\left(0 \mathrm{HU}, 0.093 \mathrm{~cm}^{-1}\right)$, and $\left(1,326 \mathrm{HU}, 0.172 \mathrm{~cm}^{-1}\right)$ (15). For voxels outside the CT field of view, the attenuation coefficients derived from the Dixon sequence were used. For each participant dataset, 2 reconstructed PET images were generated, one based on Dixon MRAC and the other based on CTAC. It should be emphasized that exactly the same reconstruction algorithm was used for both MRAC and CTAC, the only difference was the source of the attenuation map.

\section{Image Analysis}

Quantitative image analysis was performed using our standard amyloid imaging analysis protocol reported previously (24) using FreeSurfer (Martinos Center for Biomedical Imaging) regions of interest (ROIs). FreeSurfer (version 5.1) (27) was used to automatically segment participant MPRAGE data. PET to MR registration was performed using a vector gradient method (26). Regional intensity for each ROI was extracted by resampling the PET data to patient MR space to minimize any effects due to patient motion between the acquisition of the MPRAGE and PET. The target-to-reference ratio (SUVR) was calculated using the entire cerebellum as reference (28). In addition to individual FreeSurfer regions, mean cortical SUVR (MCSUVR) was also assessed using the same set of selected regions that defines mean cortical binding potential for PiB imaging $(24,29)$.

\section{Clinical Assessment}

To determine the impact of MRAC on clinical diagnosis based on amyloid imaging, the 80 reconstructed AV45 PET images (40 from MRAC and 40 from CTAC) were de-identified and randomized before being sent to an experienced nuclear medicine physician and 2 nuclear medicine residents for clinical assessment without prior knowledge about this cohort and the reconstruction method for individual image. For both the MRAC and the CTAC PET images, the same MPRAGE MRI dataset for the subject was provided for fusion and anatomic correlation with the PET data. Clinical interpretation of the florbetapir was performed according to the manufacturer's recommendations as described in the Food and Drug Administration prescribing information (30). Briefly, florbetapir PET studies were classified as positive or negative for increased amyloid based on decreased or absent gray-white differentiation larger than a single gyrus in 2 or more regions (frontal, parietal, temporal, or occipital) of the cerebrum or focally increased activity in one or more cerebral cortical regions. The primary image review was performed in the axial plane using an inverted gray scale look-up table. In addition to rating the images as positive or negative for amyloid, the confidence in this assessment for each case was rated as high, moderate, or low. According to the Food and Drug Administration prescribing information (30) for florbetapir, the PET images for clinical evaluation should be reconstructed with AC. Although in theory it may be possible to perform visual rating based on the non-attenuationcorrected PET data, it is beyond the scope of this current study.

\section{RESULTS}

\section{Image Analysis}

Example participant images are illustrated in Figure 1. The image intensity was significantly underestimated $(P<0.0001)$ using MRAC for most regions of the brain (Fig. 2). The difference was $-4.2 \% \pm 2.1 \%,-6.2 \% \pm 2.4 \%$, and $-3.2 \% \pm 2.1 \%$ for the whole brain, cortical gray matter, and cerebral white matter, respectively. Greater underestimation was observed for the cerebellum: $-6.5 \% \pm 3.0 \%$ (cerebellar white matter) and $-8.5 \% \pm$ $3.2 \%$ (cerebellar gray matter). A small overestimation in image intensity was observed for subcortical nuclei such as the caudate $(3.0 \% \pm 2.2 \%)$ and the putamen $(1.7 \% \pm 2.0 \%)$. Large spatial variability was observed, for example, the MRAC versus CTAC intensity differences varied from $2.1 \%$ to $-15.4 \%$. Large interindividual 


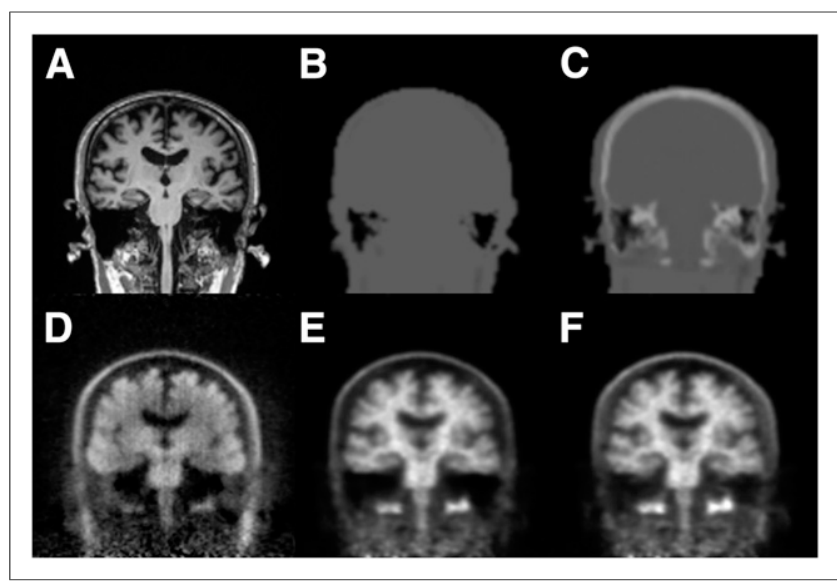

FIGURE 1. Example images of participant: T1-weighted MR (A), Dixon $\mu$-map (B), CT $\mu$-map (C), non-attenuation-corrected PET (D), PET with Dixon MRAC (E), and PET with CTAC (F).

variability also existed, for example, the MRAC versus CTAC intensity differences varied from a low of $-1.6 \%$ to a high of $-12.1 \%$ for cortical gray matter and $0.0 \%$ to $-9.7 \%$ for the whole brain.

For regional SUVR analysis using the entire cerebellum as a reference, most of the brain regions had higher SUVR using MRAC than CTAC (Fig. 3). MCSUVR measurements with MRAC were $3.6 \% \pm 3.0 \%$ higher $(P<0.0002)$ than with CTAC. The 2 measurements were strongly correlated $(r=0.99, P<0.0001)$ (Fig. 4). The Spearman rank correlation between the 2 measurements was also strong $(r=0.95, P<0.0001)$, suggesting a high concordance between the 2 . Subcortical nuclei were regions that had the largest overestimation in SUVR. For example, the thalamus, caudate, and amygdala SUVRs were overestimated by $11.1 \% \pm 3.0 \%, 11.9 \% \pm$

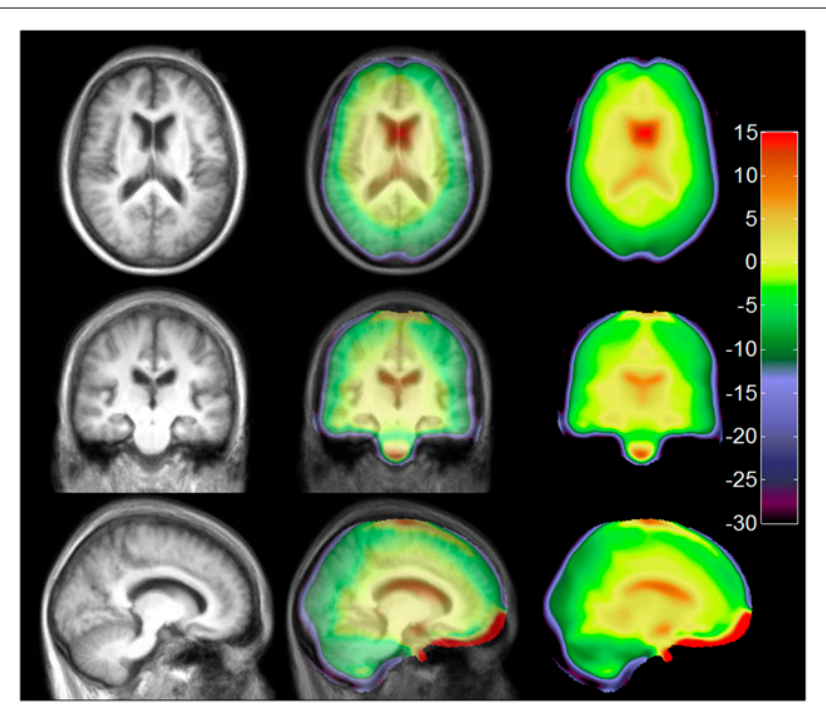

FIGURE 2. Average PET image intensity percentage difference of MRAC vs. CTAC of study cohort in atlas space. (Left) Average T1weighted MR in atlas space. (Middle) Average percentage difference map in atlas space superimposed with MR. (Right) Average percentage difference map in atlas space. Negative values indicate underestimation using MRAC against CTAC.
$3.2 \%$, and $11.9 \% \pm 3.1 \%$, respectively. Similar to raw image intensity differences, large interindividual variability also existed, for example, the difference of MCSUVR between MRAC and CTAC ranged from $-2.3 \%$ to $11.7 \%$ within our cohort.

\section{Clinical Assessment}

Clinical assessment resulted in exactly the same classification based on the 2 different AC methods: 12 of 40 participants were classified as amyloid-positive based on the clinical reads. The confidence level of the clinical rating also appears to be similar. For the CTAC-based reconstruction, 1 case was rated with low confidence, 8 were rated with moderate confidence, and the rest were rated with high confidence. For the MRAC-based reconstruction, 2 cases were rated with low confidence, 8 were rated with moderate confidence, and the rest were rated with high confidence. In general, MRAC PET data can be visually differentiated from the CTAC data by looking for signal loss in the skull base and temporal bone; however, this did not affect the physician's ability to perform clinical diagnosis. The clinical assessment from the 2 nuclear medicine residents had small discrepancies between the $2 \mathrm{AC}$ methods: 2 of $40(5 \%)$ and 5 of $40(12.5 \%)$. The raters tended to report lower confidence for those discrepant cases. The clinical assessments were different for $18(22.5 \%)$ datasets between another 2 raters and evenly distributed between MRAC and CTAC datasets; the assessments were different for $15(18.75 \%)$ datasets for 2 raters, and 9 mismatches were from CTAC datasets whereas the rest of the 6 cases were from MRAC; and the assessments were different for $13(16.25 \%)$ datasets for 2 raters, with 6 mismatches from CTAC and 7 from MRAC.

\section{DISCUSSION}

Consistent with a previous report (19), a strong spatially varying bias of PET activity was observed in neuroimaging studies using a PET/MR hybrid scanner with AC not accounting for bones. The

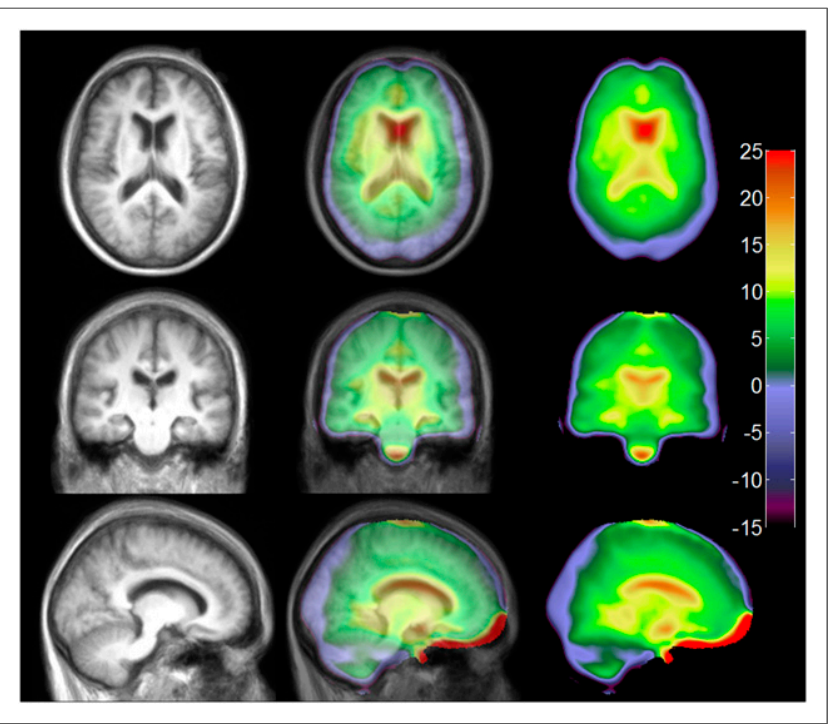

FIGURE 3. Average SUVR percentage difference of MRAC vs. CTAC of study cohort in atlas space. (Left) Average T1-weighted MR in atlas space. (Middle) Average percentage difference map in atlas space superimposed with MR. (Right) Average percentage difference map in atlas space. Negative values indicate underestimation using MRAC against CTAC. 


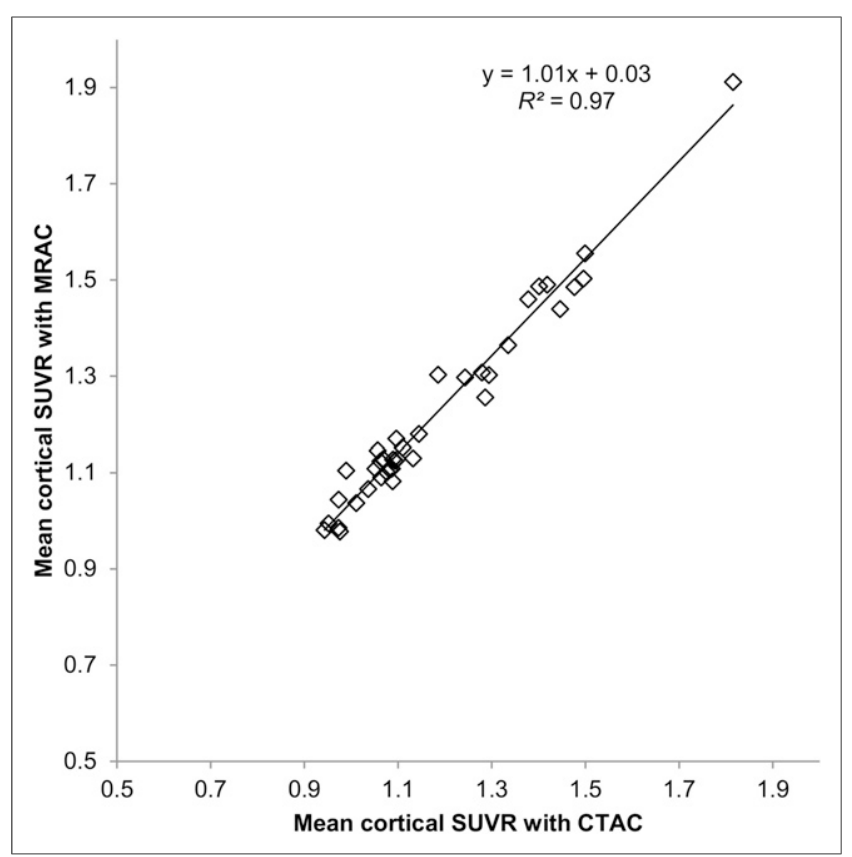

FIGURE 4. Comparison of mean cortical SUVR measurements obtained using MRAC vs. CTAC. Regions included in mean cortical SUVR was defined previously (24).

negative bias was most prominent near the surface of the brain close to cortical bone. We also observed a small positive bias for deep brain regions, likely because the Dixon-based MRAC technique assigns a constant attenuation coefficient $\left(0.1 \mathrm{~cm}^{-1}\right)$ to brain tissue, which is greater than the average brain attenuation coefficient $\left(0.095 \mathrm{~cm}^{-1}\right)$ converted from CT measurements. Our observed underestimation of PET activity was smaller than what was reported by Andersen et al. (19). In their work, a different approach was used to convert CT Hounsfield units to PET attenuation maps (31). On the basis of their approach, the estimated attenuation coefficients for brain tissue would be approximately $0.1 \mathrm{~cm}^{-1}$, and greater underestimation would result. On the basis of the transmission scans using germanium sources, the measured attenuation coefficient for brain soft tissue is approximately 0.095 $\mathrm{cm}^{-1}$ (Su, unpublished data, November 2014). In a previous study comparing CTAC against germanium transmission scan-based AC, the CTAC resulted in approximately $5 \%$ overestimation in PET activity in the cerebellum and temporal lobe, although part of the discrepancy was attributed to the fact that instead of using measured attenuation maps for $\mathrm{AC}$, the transmission scan using germanium sources was segmented and each tissue class was assigned a fixed value, while the $\mathrm{CT}$ values were transformed to attenuation coefficients in a continuous map (32). Therefore, even though in this and many other studies $(10,12,18,19)$, CTAC was used as the gold standard AC technique, one should keep in mind the variability in CTAC implementations as well as the difference between CTAC and measured attenuation maps using $511-\mathrm{keV}$ photon sources.

In addition to assessing the quantitative accuracy of measured PET activities, we also evaluated the accuracy of SUVR measurements commonly used in amyloid PET imaging and found overestimation of most brain regions. This is due to the fact that the reference region commonly used in amyloid imaging analysis, that is, the cerebellum, is one of the most underestimated regions in absolute PET activity. Because most of the brain regions were less underestimated, the SUVR measurement, which was a ratio of target region over reference region, became overestimated. Although the regional SUVR measurements were significantly different between MRAC- and CTAC-based results, the 2 sets of measurements are strongly correlated across subjects. Therefore, it is possible to covert MRAC-based SUVR measurement to CTAC-based SUVR using a linear model. With such a model, measurements obtained using the PET/MR scanner can be treated as just another PET scanner and using similar strategies to harmonize scanner differences as in previous and ongoing studies involving multiple PET scanners (33-35). Precautions have to be made, however, if the spatial pattern of amyloid deposition is of interest due to the spatially varying impact of MRAC.

Our comparison of clinical assessment for amyloid PET imaging resulted in consistent clinical rating as amyloid-positive or amyloid-negative between the 2 methods of AC. The variability due to $\mathrm{AC}$ was lower than interrater variability, and more experienced raters generated identical ratings regardless of the attenuation methods. This is highly encouraging for the adoption of a PET/ MR hybrid scanner as a clinical tool for amyloid PET imaging given the reduction in radiation doses and potential reduction in total imaging time by simultaneously acquiring PET and MR images.

It should be pointed out that the MRAC approach we evaluated in this study is specific to the Siemens Biograph mMR scanner. The Philips Ingenuity TF system (7) implemented an MR-based approach that segments the MR image into air, lung, and soft tissue and does not account for bones. In a comparison study against measured attenuation map, region-dependent underestimation of up to $20 \%$ can occur for the Philips version of MRAC (36). For the Signa PET/MR system (8), we did not find systematic evaluation of MRAC in the literature, likely due to the short time since its release. However, by default it uses an MRAC technique (8) similar to the Dixon-based approach implemented on the Siemens scanner. Therefore, an impact similar to what we observed in this study for quantitative accuracy is expected. An atlas-based approach was reported as a special case option for head scans on the GE Healthcare scanner, which may reduce the quantitative deviation in measured activity concentration because it incorporates the skull into the $\mathrm{AC}(8)$.

In this study, we evaluated the impact of attenuation map differences on the quantitative accuracy of reconstructed PET images. Attenuation maps not only affect AC, but also affect scatter correction. Therefore, the difference we observed was a combined effect of both attenuation and scatter correction. As shown in Supplemental Figure 1 (supplemental materials are available at http://jnm.snmjournals.org), the primary cause of the quantitative differences between MRAC- and CTAC-based PET images were due to the AC. Although scatter correction does change the distribution of image intensity, the change appears to be similar for both MRAC and CTAC data.

\section{CONCLUSION}

Quantitative differences were observed in this comparison study of MRAC and CTAC as has been reported in the literature before (18-20). However, this quantitative difference can be alleviated by a linear model because of the strong correlation between the 2 types of measurements. Although MR-based attenuation will result in changes in the observed spatial patterns of the PET activity distribution, the AC method used did not make a difference for clinical interpretation of studies as positive or negative for amyloid. These results suggest that MRAC PET images obtained directly 
from the scanner can be used for clinical diagnosis without the need of additional corrections.

\section{DISCLOSURE}

The costs of publication of this article were defrayed in part by the payment of page charges. Therefore, and solely to indicate this fact, this article is hereby marked "advertisement" in accordance with 18 USC section 1734 . Funding for this study was provided by The Charles F. and Joanne Knight Alzheimer's Research Initiative, NIH/NIA P50 AG05681, NIH/NIA P01AG026276, NIH/NIA P01AG003991, NIH/NIA P50 AG05681, NIH 5P30NS048056, and NIH 2UL1TR000448. Avid Radiopharmaceuticals (a wholly owned subsidiary of Eli Lilly) provided the florbetapir tracer for the study and partial funding support of the scan acquisitions. Dr. Priatna is a full-time employee of Siemens Medical Solutions, USA. The industry sponsors had no role in the design and execution of this study. No other potential conflict of interest relevant to this article was reported.

\section{REFERENCES}

1. Heiss WD. The potential of PET/MR for brain imaging. Eur J Nucl Med Mol Imaging. 2009;36(suppl 1):S105-S112.

2. Zaidi H, Del Guerra A. An outlook on future design of hybrid PET/MRI systems. Med Phys. 2011;38:5667-5689.

3. Catana C, Guimaraes AR, Rosen BR. PET and MR imaging: the odd couple or a match made in heaven? J Nucl Med. 2013;54:815-824.

4. Pichler BJ, Kolb A, Nagele T, Schlemmer HP. PET/MRI: paving the way for the next generation of clinical multimodality imaging applications. $\mathrm{J} \mathrm{Nucl} \mathrm{Med}$. 2010;51:333-336.

5. Catana C, Drzezga A, Heiss WD, Rosen BR. PET/MRI for neurologic applications. J Nucl Med. 2012;53:1916-1925.

6. Su Y, Arbelaez AM, Benzinger TL, et al. Noninvasive estimation of the arterial input function in positron emission tomography imaging of cerebral blood flow. J Cereb Blood Flow Metab. 2013;33:115-121.

7. Zaidi $\mathrm{H}$, Ojha N, Morich $\mathrm{M}$, et al. Design and performance evaluation of a whole-body Ingenuity TF PET-MRI system. Phys Med Biol. 2011;56:30913106.

8. Delso G, Furst S, Jakoby B, et al. Performance measurements of the Siemens mMR integrated whole-body PET/MR scanner. J Nucl Med. 2011;52:19141922.

9. Iagaru A, Mittra E, Minamimoto R, et al. Simultaneous whole-body time-offlight ${ }^{18}$ F-FDG PET/MRI: a pilot study comparing SUVmax with PET/CT and assessment of MR image quality. Clin Nucl Med. 2015;40:1-8.

10. Navalpakkam BK, Braun H, Kuwert T, Quick HH. Magnetic resonance-based attenuation correction for PET/MR hybrid imaging using continuous valued attenuation maps. Invest Radiol. 2013;48:323-332.

11. Martinez-Möller A, Souvatzoglou M, Delso G, et al. Tissue classification as a potential approach for attenuation correction in whole-body PET/MRI: evaluation with PET/CT data. J Nucl Med. 2009;50:520-526.

12. Keereman V, Fierens Y, Broux T, De Deene Y, Lonneux M, Vandenberghe S. MRI-based attenuation correction for PET/MRI using ultrashort echo time sequences. J Nucl Med. 2010;51:812-818.

13. Bailey DL. Transm scanning in emission tomography. Eur J Nucl Med. 1998;25:774-787.
14. Bai CY, Shao L, Da Silva AJ, Zhao Z. A generalized model for the conversion from CT numbers to linear attenuation coefficients. IEEE Trans Nucl Sci. 2003;50:1510-1515.

15. Kamel E, Hany TF, Burger C, et al. CT vs ${ }^{68} \mathrm{Ge}$ attenuation correction in a combined PET/CT system: evaluation of the effect of lowering the CT tube current. Eur J Nucl Med Mol Imaging. 2002;29:346-350.

16. Kinahan PE, Townsend DW, Beyer T, Sashin D. Attenuation correction for a combined 3D PET/CT scanner. Med Phys. 1998;25:2046-2053.

17. Hofmann M, Bezrukov I, Mantlik F, et al. MRI-based attenuation correction for whole-body PET/MRI: quantitative evaluation of segmentation- and atlas-based methods. J Nucl Med. 2011;52:1392-1399.

18. Akbarzadeh A, Ay MR, Ahmadian A, Alam NR, Zaidi H. MRI-guided attenuation correction in whole-body PET/MR: assessment of the effect of bone attenuation. Ann Nucl Med. 2013;27:152-162.

19. Andersen FL, Ladefoged CN, Beyer T, et al. Combined PET/MR imaging in neurology: MR-based attenuation correction implies a strong spatial bias when ignoring bone. Neuroimage. 2014;84:206-216.

20. Samarin A, Burger C, Wollenweber SD, et al. PET/MR imaging of bone lesions: implications for PET quantification from imperfect attenuation correction. Eur J Nucl Med Mol Imaging. 2012;39:1154-1160.

21. Klunk WE, Engler H, Nordberg A, et al. Imaging brain amyloid in Alzheimer's disease with Pittsburgh Compound-B. Ann Neurol. 2004;55:306-319.

22. Wong DF, Rosenberg PB, Zhou Y, et al. In vivo imaging of amyloid deposition in Alzheimer disease using the radioligand ${ }^{18} \mathrm{~F}-\mathrm{AV}-45$ (florbetapir [corrected] F 18). J Nucl Med. 2010;51:913-920.

23. Mintun MA, Larossa GN, Sheline YI, et al. $\left[{ }^{11} \mathrm{C}\right] \mathrm{PIB}$ in a nondemented population: potential antecedent marker of Alzheimer disease. Neurology. 2006;67: $446-452$.

24. Su Y, D'Angelo GM, Vlassenko AG, et al. Quantitative analysis of PiB-PET with FreeSurfer ROIs. PLoS One. 2013;8:e73377.

25. Morris JC. The Clinical Dementia Rating (CDR): current version and scoring rules. Neurology. 1993;43:2412-2414.

26. Rowland DJ, Garbow JR, Laforest R, Snyder AZ. Registration of $\left[{ }^{18}\right.$ F]FDG microPET and small-animal MRI. Nucl Med Biol. 2005;32:567-572.

27. FreeSurfer. Harvard.edu website. https://surfer.nmr.mgh.harvard.edu/fswiki. 2014. Accessed March 1, 2016.

28. Landau SM, Mintun MA, Joshi AD, et al. Amyloid deposition, hypometabolism, and longitudinal cognitive decline. Ann Neurol. 2012;72:578-586.

29. Su Y, Benzinger T, Vlassenko A, Blazey T, Vora S, Mintun M. Quantification of amyloid deposition with partial volume correction [abstract]. J Nucl Med. 2011;52(suppl 1):545.

30. Florbetapir prescribing information. Food and Drug Administration website. http://www.accessdata.fda.gov/drugsatfda_docs/label/2012/202008s000lbl.pdf. Accessed March 1, 2016.

31. Carney JP, Townsend DW, Rappoport V, Bendriem B. Method for transforming CT images for attenuation correction in PET/CT imaging. Med Phys. 2006;33: 976-983.

32. Nakamoto Y, Osman M, Cohade C, et al. PET/CT: comparison of quantitative tracer uptake between germanium and CT transmission attenuation-corrected images. J Nucl Med. 2002;43:1137-1143.

33. Joshi A, Koeppe RA, Fessler JA. Reducing between scanner differences in multicenter PET studies. Neuroimage. 2009;46:154-159.

34. Morris JC, Aisen PS, Bateman RJ, et al. Developing an international network for Alzheimer research: The Dominantly Inherited Alzheimer Network. Clin Investig (Lond). 2012;2:975-984.

35. Weiner MW, Aisen PS, Jack CR Jr., et al. The Alzheimer's disease neuroimaging initiative: progress report and future plans. Alzheimers Dement. 2010;6:202-211.

36. Schramm G, Langner J, Hofheinz F, et al. Quantitative accuracy of attenuation correction in the Philips Ingenuity TF whole-body PET/MR system: a direct comparison with transmission-based attenuation correction. MAGMA. 2013;26: $115-126$. 